\title{
BMJ Open Early intervention services for non- psychotic mental health disorders: a scoping review protocol
}

\author{
Katie Richards (D) , ${ }^{1}$ Amelia Austin (D) , ${ }^{1}$ Karina Allen, ${ }^{1,2,3}$ Ulrike Schmidtt ${ }^{1,2}$
}

\begin{abstract}
To cite: Richards $K$, Austin A, Allen $\mathrm{K}$, et al. Early intervention services for non-psychotic mental health disorders: a scoping review protocol. BMJ Open 2019;9:e033656. doi:10.1136/ bmjopen-2019-033656

- Prepublication history for this paper is available online. To view these files, please visit the journal online (http://dx.doi. org/10.1136/bmjopen-2019033656).
\end{abstract}

Received 15 August 2019 Revised 02 0ctober 2019 Accepted 01 November 2019

Check for updates

(C) Author(s) (or their employer(s)) 2019. Re-use permitted under CC BY. Published by BMJ.

${ }^{1}$ Psychological Medicine, King's College London, London, UK

${ }^{2}$ Eating Disorder Outpatients

Service, South London and Maudsley Mental Health NHS Trust, London, UK

${ }^{3}$ School of Psychological Science, The University of Western Australia, Perth, Western Australia, Australia

Correspondence to

Katie Richards;

katie.richards@kcl.ac.uk

\begin{abstract}
Introduction Worldwide mental health disorders are associated with a considerable amount of human suffering, disability and mortality. Yet, the provision of rapid evidence-based care to mitigate the human and economic costs of these disorders is limited. The greatest progress in developing and delivering early intervention services has occurred within psychosis. There is now growing support for and calls to extend such approaches to other diagnostic groups. The aim of this scoping review is to systematically map the emerging literature on early intervention services for non-psychotic mental health disorders, with a focus on outlining how services are structured, implemented and scaled.
\end{abstract}

Methods and analysis The protocol was developed using the guidance for scoping reviews in the Joanna Briggs Institute manual and the Preferred Reporting Items for Systematic Reviews and Meta-Analyses extension for scoping reviews checklist. A systematic search for published and unpublished literature will be conducted using the following databases: (1) MEDLINE, (2) PsycINFO, (3) HMIC, (4) EMBASE and (5) ProQuest. To be included, documents must describe and/or evaluate an early intervention service for adolescents or adults with a non-psychotic mental health disorder. There will be no restrictions on publication type, study design and date. Title and abstract, and full-text screening will be completed by one reviewer, with a proportion of articles screened in duplicate. Data analysis will primarily involve a qualitatively summary of the early intervention literature, the characteristics of early intervention services and key findings relating to their evaluation and implementation. Ethics and dissemination The synthesis of published and unpublished articles will not require ethical approval. The results of this scoping review will be published in a peer-reviewed journal and disseminated via social media, conference presentations and other knowledge translation activities.

\section{INTRODUCTION}

Early intervention is widely perceived as beneficial in medicine and refers to the early detection and initiation of stage-specific treatment. ${ }^{1}$ Proactive treatments matched to the stage of illness can limit or even avert unfavourable outcomes, reducing the need for costly and more invasive treatments in the future. ${ }^{23}$ Despite such promise, early

\section{Strengths and limitations of this study}

This scoping review will provide a comprehensive overview of both published and unpublished literature for the emerging research field of early intervention services for non-psychotic mental health disorders.

- The review will be conducted according to the standardised methodology outlined in the Joanna Briggs Institute manual and using the Preferred Reporting Items for Systematic Reviews and Meta-Analyses checklist for scoping reviews.

- Part of the screening and charting process will be completed in duplicate to ensure reliability of these methods.

- Only articles written in English, German, French and Spanish will be included, the review may, therefore, be biassed.

intervention approaches have been slow to gain momentum in mental health. ${ }^{45}$ Mental illnesses are a major contributor to mortality and disability worldwide, particularly for young people. ${ }^{6-8}$ The typical age of onset for mental disorders is adolescence and early adulthood (12-30 years), a period of marked social, psychological and biological change. ${ }^{910}$ A delay in or lack of access to effective treatments during this time could disrupt key developmental milestones and have long-lasting effects on health, social and occupational trajectories. ${ }^{11}$

Service provision does not match the topography of onset or burden of disease associated with mental disorders, even in relatively welldeveloped health systems. ${ }^{12}$ Globally, access to evidence-based care is poor, and even for those that do access it, this is often after lengthy delays. ${ }^{13-15}$ The duration of untreated illness (DUI), defined as the period between the onset of psychiatric disorder and the initiation of treatment, ranges from 1 to 2 years for psychosis to 10 years for obsessive-compulsive disorder (OCD) ${ }^{16-19}$ Over time, mental disorders can become more entrenched through functional deterioration, neuroadaptation 
and habitual behaviour patterns. ${ }^{20-23}$ Indeed, a longer DUI is associated with worse symptomatic and functional outcomes, and a lower treatment response across diagnostic groups. ${ }^{19}{ }^{24-27}$ More worryingly, young people, the group at highest risk for psychiatric difficulties, tend to have the worst access to timely care. ${ }^{13} 1828-30$

Together, such findings provide a compelling case for establishing early intervention services that match the developmental needs and symptomatic profile of individuals with recent-onset mental disorders. ${ }^{414}$ The greatest strides in early intervention have been made within psychosis. Over the past 30 years, early intervention for psychosis (EIP) has gained tremendous support from researchers and healthcare professionals worldwide. ${ }^{14}$ EIP services have two fundamental aims: to reduce the duration of untreated psychosis, and to provide evidencebased, stage-specific treatment. ${ }^{31}$ EIP services use a clinical staging approach to map the extent of illness progression from early presymptomatic risk to severe and enduring, enabling a prevention orientated framework that matches the intensity of treatment to the level of need. ${ }^{32} 33$ A comprehensive body of high-quality research shows that compared with standard care, multicomponent EIP services are associated with a reduction in symptom severity, relapse rates and hospitalisation risk, as well as improved global functioning and quality of life. ${ }^{34}$ Moreover, consistent evidence suggests that EIP services are a cost-effective alternative to standard care. ${ }^{35}$ There has been a recent surge in papers calling for early intervention approaches to be broadened to other diagnostic groups, including major depression, ${ }^{36}$ OCD ${ }^{22}$ eating disorders ${ }^{37}$ and bipolar disorder. ${ }^{38}$ Preliminary evidence from services for recent-onset eating and mood disorders demonstrate significant improvements in symptoms, reduced hospital (re)admissions, and most importantly, high levels of patient satisfaction. ${ }^{39-42}$

The utility of focusing exclusively on discrete diagnostic categories in the delivery of early intervention specifically, and mental healthcare more generally has, however, been questioned. $^{32}{ }^{43}$ The early stages of mental disorder are often characterised by fluctuating patterns of specific and non-specific subthreshold symptoms, diagnostic instability and comorbidity. ${ }^{45}$ A single-disorder focus could result in these earlier presentations of illness being excluded. ${ }^{46}$ A transdiagnostic approach, consistent with evidence for pluripotent models of clinical staging, has been put forward as a necessary solution to address this problem. ${ }^{32} 434748$ The recognition of the need to broaden the early intervention paradigm has led to the development of several integrated youth mental health hubs. ${ }^{490}$ These hubs act as entry-level services for young people irrespective of diagnosis, and typically provide a comprehensive package of low-intensity mental, physical and social care support in community settings. Young people tend to rate these services positively and between $52 \%$ and $68 \%$ experience improvements in symptoms and functioning. However, a proportion of individuals with more severe symptoms do not seem to benefit from these services and rigorous outcome research for youth hubs is limited. ${ }^{50} 51$

Although the role of early intervention in reducing distress and functional impairment seems obvious, the evidence-base for these services is incomplete and much more work needs to be done. ${ }^{1422}$ There is limited prospective evidence evaluating the utility of these services for non-psychotic disorders, it is unclear to what extent the findings from psychosis would translate to other diagnostic groups. There is also a lack of research evaluating the feasibility or the implementation processes of services in clinical settings. ${ }^{51}$ Moreover, even within psychosis, further research is needed to determine how long EIP services should be provided, whether it is the reduction in DUI or other components of EIP services that account for the improved outcomes, and whether outcomes would be similar with other service structures and models. ${ }^{52}{ }^{53}$ An ever-growing population, accompanied by reducing health budgets, creates an environment where only services that demonstrate effectiveness, economic viability and sustainability receive funding. ${ }^{54}$ It is, therefore, imperative to develop a rigorous evidence base to refine, adapt and evaluate early intervention services for non-psychotic disorders, with a particular focus on identifying the "active ingredients" of such services and the most effective methods for widespread scaling and implementation.

The primary objective of this review is to provide a baseline characterisation of the differing ways in which early intervention services are structured and implemented for non-psychotic mental health disorders. The emerging literature for non-psychotic disorders is heterogeneous and dispersed, with distinct streams of research developing in disciplinary silos. The aim of this review is to draw together these streams to facilitate collaboration and cross-disciplinary learning and discourse. By synthesising the field and highlighting commonalities and differences, we hope that a broad set of common principles for early intervention services will emerge. This review, in conjunction with reviews in psychosis, will help set the stage for a more unified approach to expanding and refining early intervention services for psychiatric disorders. Here, we focus exclusively on disorders that tend to emerge in adolescence and adulthood rather than in childhood. Neurodevelopmental disorders typically use a very different approach to early intervention than adolescent-onset and adultonset disorders (eg, intervening in infancy). ${ }^{55} \mathrm{~A}$ scoping review methodology was selected for this review as early intervention is an emerging, dispersed and heterogeneous research area and is therefore not amenable to the narrower aims of a traditional systematic review. ${ }^{567}$ Given that this is a relatively new research area, we sought to map all the available evidence within this field rather than only the best available evidence (eg, randomised controlled trials)..$^{58}$ 
Table 1 MEDLINE search strategy

\begin{tabular}{|c|c|c|}
\hline & Query & Results \\
\hline \#1 & exp Early Medical Intervention [MeSH term)/ or (early intervention* or early-intervention*).tw & 19623 \\
\hline \#2 & 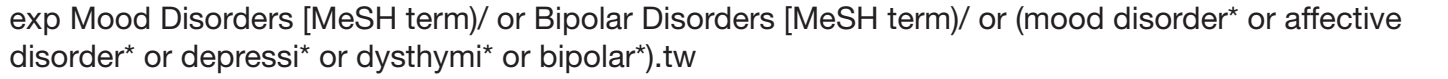 & 453041 \\
\hline \#3 & \#1 AND \#2 & 1616 \\
\hline \#4 & $\begin{array}{l}\text { exp Anxiety Disorders [MeSH term)/ or (anxiety disorder* or neurotic disorder* or agoraphobi* or } \\
\text { obsessive-compulsive disorder }{ }^{\star} \text { or OCD or panic disorder* or phobic disorder* or post-traumatic stress } \\
\text { disorder or post traumatic stress disorder* or PTSD or generalised anxiety disorder or social phobia). } \\
\text { tw }\end{array}$ & 119604 \\
\hline
\end{tabular}

$\begin{array}{ll}\text { \#5 } & \text { \#1 AND \#4 } \\ \text { \#6 } & \begin{array}{l}\text { exp “Feeding and Eating Disorders" [MeSH term)/ or (eating disorder* or anorexi* or bulimi* or binge- } \\ \text { eating* or binge eating* or (eating disorder not otherwise specified) or EDNOS or (other specified }\end{array}\end{array}$
feeding or eating disorder) or OSFED).tw

$\begin{array}{ll}\text { \#7 } & \text { \#1 AND \#6 } \\ \text { \#8 } & \text { exp Substance-Related Disorders [MeSH term)/ or exp “Disruptive, Impulse Control, and Conduct } 295108\end{array}$

Disorders" [MeSH term)/ or (((substance-related or alcohol or opioid or morphine or marijuana or heroin or cocaine or amphetamine or cannabis) adj1 (disorder* or illness* or dependence or abuse or misuse)) or (impulse control disorder ${ }^{\star}$ ) or conduct disorder ${ }^{\star}$ or fire setting behaviour or gambling or trichotillomania).tw

\begin{tabular}{|c|c|c|}
\hline \#9 & \#1 AND \#8 & 924 \\
\hline \#10 & $\begin{array}{l}\text { exp Somatoform Disorders [MeSH term)/ or (somatoform or somatoform disorder or somati\#ation or } \\
\text { body dysmorphi* or conversion disorder* or hypochondri*).tw }\end{array}$ & 25487 \\
\hline \#12 & 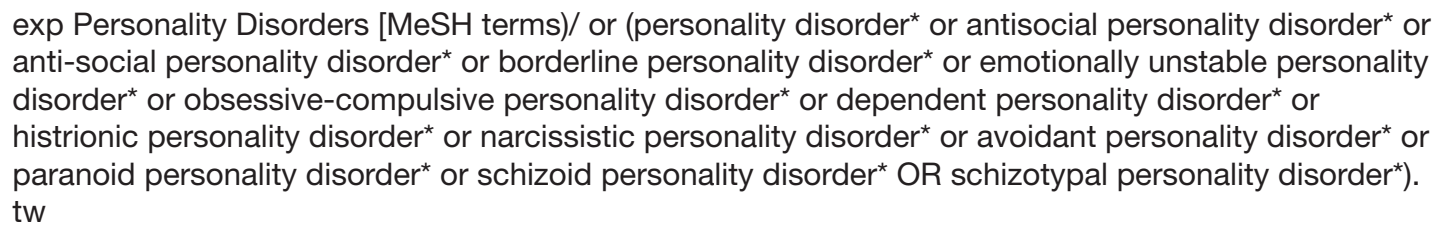 & 47019 \\
\hline$\# 13$ & \#1 AND \#12 & 208 \\
\hline
\end{tabular}

\section{RESEARCH QUESTIONS}

1. What is the extent, range and nature of the literature on early intervention services for adolescents and adults with non-psychotic mental health disorders?

2. What are the characteristics of early intervention services and care pathways?

a. Are there any similarities and/or differences across early intervention services provided for each diagnosis and transdiagnostically?

3. Are there any factors that influence the implementation of early intervention services (ie, barriers and facilitators to implementation)?

4. Do early intervention services reduce DUI, improve the course and outcome of mental disorders or minimise the disruption to psychosocial development and function?

\section{METHODS AND ANALYSIS}

The Preferred Reporting Items for Systematic Reviews and Meta-Analyses extensionfor scoping reviews (PRISMA-ScR) checklist, ${ }^{57}$ and the scoping review framework outlined in the Joanna Briggs Institute (JBI) Reviewer's Manual ${ }^{59}$ were used to guide the development of this protocol.

\section{Eligibility criteria}

Documents will be included if they: (1) Describe and/ or evaluate an early intervention service for nonpsychotic mental health disorders (concept) based in any type of healthcare facility (ie, hospitals, day services and community settings) and in any geographical area (context). Here, early intervention refers to a structured programme of care delivered by a stand-alone team or teams integrated into mental health services that provide treatment for individuals with recent-onset subthreshold or threshold disorders. The level of care can vary from low-intensity techniques of signposting, psychoeducation and self-help resources all the way through to specialised multidisciplinary teams and complex high intensity interventions; (2) Describe and/or evaluate an early intervention service for adolescents $(\geq 10-17$ years) or adults ( $>18$ years) with a recent-onset subthreshold or threshold mood disorder, anxiety disorder, eating disorder, personality disorder, impulse control or substance use disorder, and/or somatoform disorder (types of participants). 
Table 2 Draft data charting form

\section{Data item Description of item}

Document details

Type of document The type of document can include but will not be limited to published or unpublish primary research, any type of review, protocols, theoretical paper, guidelines, opinion pieces, editorials and expert consensus papers.

\begin{tabular}{ll} 
Author(s) & List of authors \\
Year of publication & Year of publication \\
\hline Title & Title of document \\
\hline Journal & The title of the scientific journal (for published documents only) \\
\hline Country of origin & Country where the document originates \\
\hline Aim/purpose of & Summary of the aim/purpose of the document
\end{tabular}

document

$\begin{array}{ll}\text { Study design } & \begin{array}{l}\text { For published or unpublished research papers, the design of the study as reported in the paper. } \\ \text { Includes but is not limited to randomised controlled trials, pre-post design, historical controlled trial, } \\ \text { prospective or retrospective cohort studies, cross-sectional and case series/study. }\end{array} \\ \text { Study methodology } & \text { The methodological framework: qualitative, quantitative or mixed methods. }\end{array}$

\section{Characteristics of early intervention service}

Name of service The name of the early intervention service/programme.

Year established The year the early intervention service was established.

Location The country and region in which the early intervention service was implemented.

Population The population for which the service was designed for. This item will include details such as age, diagnosis, duration of illness and illness severity.

Setting The physical setting in which the early intervention service is based. This includes but is not limited to community centres, primary care, outpatient clinics and inpatient wards. Early intervention services can occupy more than one of these settings.

Service providers A description of who provides the service and their role, includes but is not limited to social workers, youth workers, peer support workers, nurses, clinical or counselling psychologists and psychiatrists.

Service structure/ A description of the service structure and administrative processes includes but is not limited to process $\quad$ 'service within a service' models, stand-alone multidisciplinary team models, 'hub' and 'spoke' models, and process variables such as specific wait time targets.

Access to service Methods for accessing the early intervention service, includes but is not limited to active engagement and outreach through schools, colleges and youth clubs, referral from primary care, self-referral and drop-in.

Services and interventions

A description of the types of services and interventions provided, includes but is not limited to psychoeducation, online self-help and self-management support, psychological therapies (eg, CBT, brief therapy), sexual health and family planning, health promotion, social services, peer support, and crisis intervention and management.

Clinical staging Whether a clinical staging approach was used to inform the design, evaluation or implementation of the service.

\section{Outcome Research}

Participants Details related to the participants included in the study. This will include information related to sample size, diagnosis, age, sex and inclusion/exclusion criteria.

Comparator data or Description of comparator data or the care provided to a control group.

standard care

Outcomes and time Description of the qualitative and quantitative outcomes and the time points of data collection. This points will include standardised clinical assessments, and self-report measures as well as implementation outcomes, such as measures of acceptability, feasibility, adoption, fidelity and sustainment.

Key results/findings An outline of the key results and findings reported in the document. This includes quantitative outcomes such as changes in symptoms, engagement and patient satisfaction, as well as qualitative outcomes, such as, descriptions of barriers and facilitators to implementation.

CBT, cognitive-behavioural therapy. 
Table 3 Summary of reach, effectiveness, adoption, implementation and maintenance framework criteria

Reach (participant representativeness)
The representativeness of individuals enrolled in the study to the characteristics of the intended population.

$1=$ Limited generalisability: highly selected subsample that is not typical of the intended population, high number of exclusionary criteria, and/or a recruitment strategy that is likely to result in a biassed sample.

2=Moderately generalisable: participants match intended population on key characteristics (eg, sex/ gender, diagnosis, age), but are still a selected subsample due to exclusion criteria and recruitment strategies.

3=Generalisable: participants are typical of the intended population, limited or no exclusion criteria and/or recruitment strategies is not selective and are unlikely to result in a biassed sample.

$\begin{array}{ll}\text { Effectiveness } & \text { Measured outcomes are important and meaningful to all stakeholders involved, including potential } \\ \text { (outcome } & \text { negative effects, quality of life and economic outcomes. } \\ \text { representativeness) } & \text { 1=Limited generalisability: primary outcomes restricted to an estimate of the overall effect of the } \\ & \text { intervention on a single metric of health, limited attention to process outcomes, quality of life, patient } \\ & \text { and staff satisfaction, patient engagement, unintended harms, or functional rehabilitation. } \\ & \text { 2=Moderate generalisability: primary outcomes focus on overall effect of intervention on health, some } \\ \text { inclusion of measures that are meaningful to stakeholders or process outcomes. } & \\ \text { 3=Generalisable outcomes: primary outcomes include mix of impact of intervention on health and } \\ \text { outcomes that are meaningful to patients and other stakeholders (including qualitative evaluations), } \\ \text { explicit discussion around prevention of harms to participants, process outcomes, patient } \\ \text { engagement, acceptability and satisfaction. }\end{array}$

Adoption (setting representativeness)

The representativeness of settings and the individuals within those settings who deliver the programme. Implementation
(fidelity/adaptation,
and cost/feasibility)
1=Limited generalisability: highly selected settings and staff and/or only includes 'best' sites and staff, that is, well-resourced, credentialed or seasoned interventionists, many exclusion criteria; or limited information to determine context of study or intervention.

2=Moderate generalisability: intervention tested in contexts outside of 'best' sites and staff, but adoption is still limited to selected settings that are well resourced with some expertise in intervention trials.

3=Generalisable: sites and staff are randomly selected, few or no exclusion criteria and/or trialled in diverse settings.

Fidelity to the intervention and adaptations made to intervention during study/programme.

$1=$ Limited information on the implementation: no details on adaptation to local context, no details related to core element of interventions, or an evaluation of the consistency of implementation across settings staff, and patients.

2=Moderate reporting of fidelity/adaptations: core elements described but details missing, or fidelity was monitored but no details on measurement tools.

$3=$ Detailed report of modifications made, adaptations to local context, and rationale for modification, an outline of core elements and evaluation of the fidelity to core elements of the model.

The cost of the intervention in terms of time and money.

$1=$ No details on time, cost and resources, no efforts to contain costs, and use of state-of-the-art resources and procedures such that costs of intervention are likely to be high.

$2=$ Details on time, cost and resources is still limited but more than for a rating of 1 . The intervention has minimal impact on time, cost and resources.

$3=$ Explicit efforts to contain costs and to make the intervention feasible in low resource settings.

Maintenance

(sustainment)
The extent to which an intervention becomes institutionalised or part of the routine organisational practices and policies and the extent to which behaviour is sustained for more than 6 months. $1=$ Limited sustainability efforts or details of such efforts: no report of efforts to continue an intervention after the completion of study, or no reports of continued use.

2=Moderate sustainment: limited discussion regarding the sustainability of an intervention, some evidence of continued use.

3=Sustainment: long-term outcomes reported, explicit plans for handing off intervention to setting/ sites, details of methods to encourage sustainable implementation or embedding within routine organisational practices and policies or evidence of sustained use for 6 months or more.
Transdiagnostic early intervention services and early intervention services for comorbid/concurrent disorders will be included provided that at least one of the diagnoses is listed in the previous sentence; (3) Mixed child and adolescent services will be included, where feasible, only information relevant for the adolescent portion of the services will be charted and (4) All document types and study designs are eligible for inclusion: randomised 
controlled trials, non-randomised studies, observational studies, qualitative studies, reviews, ongoing trials, protocols, theoretical papers, grey literature, editorials, opinions pieces and expert consensus statements (types of studies).

Documents will be excluded if they: (1) Describe a primary prevention programme based in educational establishments, high-risk groups (eg, athletes) or in the general population, (2) Describe a parent-only intervention, (3) Describe a specific intervention (eg, type of cognitive-behavioural therapy) that is not attached to a service and (4) Primarily or only focus on early intervention for a physiological or medical condition, schizophrenia spectrum and other psychotic disorders and/or neurodevelopmental disorders.

\section{Search strategy}

A comprehensive literature search will be conducted from inception on PsycINFO, MEDLINE, EMBASE and HMIC. ProQuest databases will also be searched for grey literature (ie, conference papers and proceedings, theses, government publications). The search is completed in three stages. First, an initial limited search was conducted in MEDLINE using the terms "early intervention" and "mood disorder" or "anxiety disorder" or "eating disorder" or "personality disorder" or "impulse control disorder" or "substance use disorder" or "somatoform disorder". The initial limited search was conducted by KR in April 2019 to identify keywords and subject headings to generate a search strategy. Different combinations of keywords and subject headings were trialled in MEDLINE, and key papers from the early intervention field were used as indicators for the sensitivity of the search strategy. The preliminary search strategy was developed by KR and reviewed by AA, KA and US. An iterative process was used to balance the sensitivity and specificity. The MEDLINEspecific search strategy returns 3545 documents before deduplication and is outlined in table 1.

In the second stage, all databases will be searched using the MEDLINE search strategy. The search strategy will be tailored to each database. The search for scoping reviews are more iterative than systematic reviews, it is; therefore, feasible that as the reviewers become more familiar with the literature that additional search terms and sources may be identified. The final stage involves identifying additional articles by searching the reference lists of included articles. Studies not reported in English, German, French and Spanish will be excluded from the review during the screening and eligibility assessment. No date limits will be applied to the search. References will be imported to the EndNote X8 reference manager.

\section{Study selection process}

The title and abstract screening in the second stage of the search will be completed by one reviewer with a portion of the articles being screened in duplicate to ensure reliability $(25 \%)$. Retrieved full texts will also be screened by one reviewer with a sample of full-text documents $(25 \%)$ being screened in duplicate for reliability. The eligibility criteria will be applied to each document on a case-bycase basis to determine eligibility for inclusion. Discrepancies between reviewers will be resolved by discussion and if necessary other members of the review team will be consulted.

\section{Data items and charting}

A standardised data charting form developed by the study team will be used to chart the data from eligible studies (see table 2 for a description of each data item). The data charting form was developed using the template from the JBI manual and by drawing on recent reviews of youth service models. ${ }^{50}$ Each section of the data charting form was developed to address one of the four research questions. The 'Document Details' section which provides descriptive information on document type, author(s), publication date, title and aim/purpose of document will be used to evaluate the extent, nature and range of the literature on early intervention services (question 1). The second section 'Characteristics of Early Intervention Service' will address the second question as key characteristics of the services, namely the population, setting, structure and interventions used in early intervention services will be charted (question 2). The 'Outcome Research' section will be used to answer questions 3 and 4 as any data related to implementation, effectiveness or efficacy will be charted (question 3 and 4). Similar to the full-text screening, one reviewer will chart the majority of the documents with only a portion $(25 \%)$ of the documents being charted in duplicate to ensure reliability. A small selection of documents will be charted by both reviewers at the outset to ensure that there is clarity and consistency in the use of the data charting form. Where there is more than one paper on the same service model, information will be pooled across the papers to provide the most detailed description of the model and any available evidence.

\section{Critical appraisal}

The lack of critical appraisal tools in scoping reviews has been highlighted as one of the primary limitations of this knowledge synthesis method. ${ }^{60}$ Critical appraisal can facilitate the interpretation of reviews by identifying the relative strengths and weaknesses of the included articles and identifying gaps in the research field. However, formal evaluations of methodological quality for scoping reviews can be challenging given the diversity of study designs and the volume of included literature ${ }^{61}$ Given the range of study designs, a two-stage assessment of methodological quality will be conducted for this review. First, each study will be ranked using the JBI Levels of Evidence for Effectiveness from high (level 1) to low (level 5) (level 1-Experimental Designs; level 2-Quasi-experimental Designs; level 3-Observational-Analytical; level 4Observational-Descriptive; level 5-Expert Opinion and Bench Research) ${ }^{62}$ Once stratified according to the level of evidence, the quality of the studies within each stratum 
will be evaluated using the JBI Critical Appraisal tools. ${ }^{63}$ Additionally, the generalisability and real-world applicability (external validity) of the included studies will be evaluated against the domains of the reach, effectiveness, adoption, implementation and maintenance (RE-AIM) framework. A modified version of a RE-AIM framework rating system developed by Gaglio et al will be used in the current study. ${ }^{64}$ The modified rating system can be seen in table 3. Each document will be given a rating ranging from 1 (limited generalisability or no information) to 3 (generalisable/pragmatic or information to enable generalisation) on six key domains: participant representativeness, setting representativeness, outcome representativeness, fidelity/adaptation, cost/feasibility of intervention and sustainment. A narrative summary of the methodological quality will be provided alongside quantitative values for each domain of the RE-AIM framework. A portion of the included articles will be appraised in duplicate.

\section{Synthesis of results}

The search results will be reported using a flow diagram to clearly detail the review decision process, indicating the number of citations screened, duplicates removed, study selection and full texts retrieved. The characteristics of the included studies will be presented in an informative table with a narrative and quantitative (eg, frequencies) summary in text. Figures will be used to display the distribution of documents over time and across diagnoses. Descriptions of the early intervention services will be reported for each diagnostic group and transdiagnostically along with any evidence supporting the services and barriers and facilitators to implementation. An aggregated summary of early intervention services with descriptions of common themes and differences across the services will be provided. An effort will be made to identify gaps in knowledge to inform the direction of future research.

\section{Patient and public involvement}

No patients or public were involved in the development of this protocol.

\section{DISSEMINATION}

This review contributes to the growing body of research for early intervention initiatives in mental health by mapping the existing literature on early intervention services for non-psychotic mental health disorders. Through the publication of the results and dissemination via social media and conference presentations, the results will hopefully provide a timely foundation for cross-disciplinary discourse and early intervention service development and research. The results of this review may inform the design of new services and policies to support them.
Contributors All authors contributed to the development of this protocol. KR drafted the manuscript and search strategy. AA, KA and US reviewed the search strategy and the draft manuscripts. KR incorporated the feedback from the authors. All authors read and approved the final manuscript.

Funding KR and AA are supported by the Health Foundation Scaling Up programme. US is supported by a National Institute of Health Research (NIHR) Senior Investigator Award and receives salary support from the NIHR Mental Health Biomedical Research Centre at the South London and Maudsley NHS Foundation Trust and King's College London. KA is supported by the NHS Innovation Accelerator programme.

Competing interests None declared.

Patient consent for publication Not required.

Ethics approval The synthesis of existing knowledge will not require ethical approval.

Provenance and peer review Not commissioned; externally peer reviewed.

Open access This is an open access article distributed in accordance with the Creative Commons Attribution 4.0 Unported (CC BY 4.0) license, which permits others to copy, redistribute, remix, transform and build upon this work for any purpose, provided the original work is properly cited, a link to the licence is given, and indication of whether changes were made. See: https://creativecommons.org/ licenses/by/4.0/.

\section{ORCID iDs}

Katie Richards http://orcid.org/0000-0003-3826-6317

Amelia Austin http://orcid.org/0000-0002-4979-4847

\section{REFERENCES}

1 McGorry PD. Is early intervention in the major psychiatric disorders justified? Yes. BMJ 2008;337:a695.

2 Gillies CL, Abrams KR, Lambert PC, et al. Pharmacological and lifestyle interventions to prevent or delay type 2 diabetes in people with impaired glucose tolerance: systematic review and metaanalysis. BMJ 2007;334.

3 Shah C, Arthur DW, Wazer D, et al. The impact of early detection and intervention of breast cancer-related lymphedema: a systematic review. Cancer Med J 2016;5:1154-62.

4 McGorry PD, Mei C. Early intervention in youth mental health: progress and future directions. Evid Based Ment Health 2018;21:182-4.

5 McGorry PD, Ratheesh A, O'Donoghue B. Early Intervention-An implementation challenge for 21 st century mental health CareEarly Intervention-An implementation challenge for 21 st century mental health CareEditorial. JAMA Psychiatry 2018;75:545-6.

6 Patel V, Flisher AJ, Hetrick S, et al. Mental health of young people: a global public-health challenge. Lancet 2007;369:1302-13.

7 Rehm J, Shield KD. Global burden of disease and the impact of mental and addictive disorders. Curr Psychiatry Rep 2019;21:10.

8 Vigo D, Thornicroft G, Atun R. Estimating the true global burden of mental illness. Lancet Psychiatry 2016;3:171-8.

9 de Girolamo G, McGorry PD, Sartorius N, eds. Age of Onset of Mental Disorders. Switzerland: Springer International, 2019.

10 Kessler RC, Angermeyer M, Anthony JC, et al. Lifetime prevalence and age-of-onset distributions of mental disorders in the world Health organization's world mental health survey initiative. World $J$ Psychiatry 2007;6:168-76.

11 Malla A, Shah J, lyer S, et al. Youth mental health should be a top priority for health care in Canada. Can J Psychiatry 2018;63:216-22.

12 Vigo DV, Kestel D, Pendakur K, et al. Disease burden and government spending on mental, neurological, and substance use disorders, and self-harm: cross-sectional, ecological study of health system response in the Americas. Lancet Public Health 2019;4:e89-96.

13 de Girolamo G, Dagani J, Purcell R, et al. Age of onset of mental disorders and use of mental health services: needs, opportunities and obstacles. Epidemiol Psychiatr Sci 2012;21:47-57.

14 McGorry PD. Early intervention in psychosis: obvious, effective, overdue. J Nerv Ment Dis 2015;203:310-8.

15 Patel V, Saxena S, Lund C, et al. The Lancet Commission on global mental health and sustainable development. Lancet 2018;392:1553-98.

16 Albert U, Barbaro F, Bramante S, et al. Duration of untreated illness and response to Sri treatment in obsessive-compulsive disorder. Euro Psychiatry 2019;58:19-26. 
17 Altamura AC, Buoli M, Albano A, et al. Age at onset and latency to treatment (duration of untreated illness) in patients with mood and anxiety disorders: a naturalistic study. Int Clin Psychopharmacol 2010;25:172-9.

18 Dagani J, Signorini G, Nielssen O, et al. Meta-Analysis of the interval between the onset and management of bipolar disorder. Can $J$ Psychiatry 2017;62:247-58.

19 Marshall M, Lewis S, Lockwood A, et al. Association between duration of untreated psychosis and outcome in cohorts of first-episode patients: a systematic review. Arch Gen Psychiatry 2005;62:975-83.

20 Anderson KK, Voineskos A, Mulsant BH, et al. The role of untreated psychosis in neurodegeneration: a review of Hypothesized mechanisms of neurotoxicity in First-Episode psychosis. Can $J$ Psychiatry 2014:59:513-7.

21 Currin L, Schmidt U. A critical analysis of the utility of an early intervention approach in the eating disorders. J Ment Health 2005;14:611-24.

22 Fineberg NA, Dell'Osso B, Albert U, et al. Early intervention for obsessive compulsive disorder: an expert consensus statement. Eur Neuropsychopharmacol 2019;29:549-65.

23 Schaffalitzky E, Leahy D, Armstrong C, et al. 'Nobody really gets it': a qualitative exploration of youth mental health in deprived urban areas. Early Interv Psychiatry 2015;9:406-11.

24 Altamura AC, Dell'osso B, D'Urso N, et al. Duration of untreated illness as a predictor of treatment response and clinical course in generalized anxiety disorder. CNS Spectr 2008;13:415-22.

25 Altamura AC, Dell'Osso B, Berlin HA, et al. Duration of untreated illness and suicide in bipolar disorder: a naturalistic study. Eur Arch Psychiatry Clin Neurosci 2010;260:385-91.

26 Drancourt N, Etain B, Lajnef M, et al. Duration of untreated bipolar disorder: missed opportunities on the long road to optimal treatment. Acta Psychiatr Scand 2013;127:136-44.

27 Ghio L, Gotelli S, Cervetti A, et al. Duration of untreated depression influences clinical outcomes and disability. $J$ Affect Disord 2015;175:224-8.

28 Cleary A, Nixon E, Fitzgerald M. Psychological health and well-being among young Irish adults. Ir J Psychol Med 2007;24:139-44.

29 Burgess PM, Pirkis JE, Slade TN, et al. Service use for mental health problems: findings from the 2007 national survey of mental health and wellbeing. Aust N Z J Psychiatry 2009;43:615-23.

30 Weigel $A$, Rossi $\mathrm{M}$, Wendt $\mathrm{H}$, et al. Duration of untreated illness and predictors of late treatment initiation in anorexia nervosa. $J$ Public Health 2014;22:519-27.

31 McGorry PD, Edwards J, Mihalopoulos C, et al. EPPIC: an evolving system of early detection and optimal management. Schizophr Bull 1996;22:305-26.

32 Cross S, Hickie I. Transdiagnostic stepped care in mental health. Public Health Res Pr 2017;27.

33 McGorry PD, Hickie IB, Yung AR, et al. Clinical staging of psychiatric disorders: a heuristic framework for choosing earlier safer and more effective interventions. Aust N Z J Psychiatry 2006:40:616-22.

34 Correll CU, Galling B, Pawar A, et al. Comparison of early intervention services vs treatment as usual for early-phase psychosis: a systematic review, meta-analysis, and meta-regression. JAMA Psychiatry 2018;75:555-65.

35 Aceituno D, Vera N, Prina AM, et al. Cost-Effectiveness of early intervention in psychosis: systematic review. Br J Psychiatry 2019;215:388-94.

36 Davey CG, McGorry PD. Early intervention for depression in young people: a blind spot in mental health care. The Lancet Psychiatry 2019;6:267-72.

37 Schmidt U, Brown A, McClelland J, et al. Will a comprehensive, person-centered, team-based early intervention approach to first episode illness improve outcomes in eating disorders? Int J Eat Disord 2016:49:374-7.

38 Vieta E, Salagre E, Grande I, et al. Early intervention in bipolar disorder. Am J Psychiatry 2018;175:411-26.

39 Brown A, McClelland J, Boysen E, et al. The FREED project (first episode and rapid early intervention in eating disorders): service model, feasibility and acceptability. Early Interv Psychiatry 2018;12:250-7.
40 Kessing LV, Hansen HV, Hvenegaard A, et al. Treatment in a specialised out-patient mood disorder clinic $V$. standard out-patient treatment in the early course of bipolar disorder: randomised clinical trial. Br J Psychiatry 2013;202:212-9.

41 Osuch E, Vingilis E, Summerhurst C, et al. Process evaluation of a treatment program for mood and anxiety disorders among emerging adults: Preentry factors, engagement, and outcomes. Psychiatr Serv 2019;70:211-8

42 McClelland J, Hodsoll J, Brown A, et al. A pilot evaluation of a novel first episode and rapid early intervention service for eating disorders (FREED). Eur Eat Disord Rev 2018;26:129-40.

43 McGorry PD, Ratheesh A, O'Donoghue B. Early Intervention-An implementation challenge for 21 st century mental health care. JAMA Psychiatry 2018;75:545-6.

44 Iorfino F, Scott EM, Carpenter JS, et al. Clinical stage transitions in persons aged 12 to 25 years presenting to early intervention mental health services with anxiety, mood, and psychotic disorders. JAMA Psychiatry 2019;76.

45 McGorry PD, Hartmann JA, Spooner R, et al. Beyond the "at risk mental state" concept: transitioning to transdiagnostic psychiatry. World Psychiatry 2018;17:133-42.

46 Cross SPM, Hermens DF, Scott EM, et al. A clinical staging model for early intervention youth mental health services. Psychiatr Serv 2014;65:939-43.

47 Scott J, Leboyer M, Hickie I, et al. Clinical staging in psychiatry: a cross-cutting model of diagnosis with heuristic and practical value. Br J Psychiatry 2013;202:243-5.

48 McGorry P, Nelson B. Why we need a Transdiagnostic staging approach to emerging psychopathology, early diagnosis, and treatment. JAMA Psychiatry 2016;73:191-2.

49 Lee VWW, Murphy BP. Broadening the early intervention paradigm: a one stop shop for youth. Early Interv Psychiatry 2013;7:437-41.

50 Hetrick SE, Bailey AP, Smith KE, et al. Integrated (one-stop shop) youth health care: best available evidence and future directions. Medical Journal of Australia 2017;207:S5-18.

51 Settipani CA, Hawke LD, Cleverley K, et al. Key attributes of integrated community-based youth service hubs for mental health: a scoping review. Int J Ment Health Syst 2019;13:52.

52 Behan C, Masterson S, Clarke M. Systematic review of the evidence for service models delivering early intervention in psychosis outside the stand-alone centre. Early Interv Psychiatry 2017;11:3-13.

53 Fusar-Poli P, McGorry PD, Kane JM. Improving outcomes of firstepisode psychosis: an overview. World Psychiatry 2017;16:251-65.

54 Stuckler D, Reeves A, Loopstra R, et al. Austerity and health: the impact in the UK and Europe. Eur J Public Health 2017;27:18-21.

55 Cioni G, Inguaggiato E, Sgandurra G. Early intervention in neurodevelopmental disorders: underlying neural mechanisms. Dev Med Child Neurol 2016:58:61-6.

56 Peters MDJ, Godfrey CM, Khalil H, et al. Guidance for conducting systematic scoping reviews. Int J Evid Based Healthc 2015;13:141-6.

57 Tricco AC, Lillie E, Zarin W, et al. PRISMA extension for scoping reviews (PRISMA-ScR): checklist and explanation. Ann Intern Med 2018:169:467-73.

58 Murray A, Daines L, Archibald D, et al. The relationship and effects of golf on physical and mental health: a scoping review protocol. $\mathrm{Br} J$ Sports Med 2016;50:647-50.

59 Peters MDJ, Godfrey C, Mclnerney P, et al. Chapter 11: Scoping Reviews. In: Aromataris E, Munn Z, eds. Joanna Briggs Institute reviewer's manual: the Joanna Briggs Institute, 2017.

60 Pham MT, Rajić A, Greig JD, et al. A scoping review of scoping reviews: advancing the approach and enhancing the consistency. Res. Syn. Meth. 2014;5:371-85.

61 Levac D, Colquhoun H, O'Brien KK. Scoping studies: advancing the methodology. Implementation Sci 2010;5.

62 Jordan Z, Lockwood C, Aromataris E, et al. The updated JBI model for evidence-based healthcare, 2016. Available: https://joannabriggs. org/jbi-approach.html\#tabbed-nav=Levels-of-Evidence

63 The Joanna Briggs Institute. Joanna Briggs Institute critical appraisa tools, 2017. Available: https://joannabriggs.org/critical_appraisal_ tools

64 Gaglio B, Phillips SM, Heurtin-Roberts S, et al. How pragmatic is it? lessons learned using Precis and RE-AIM for determining pragmatic characteristics of research. Implementation Sci 2014;9. 